\title{
Using Visual Rhythms for Detecting Video-based Facial Spoof Attacks
}

\author{
Supplemental Material
}

\author{
Allan Pinto ${ }^{1}$, William Robson Schwartz ${ }^{2}$, Helio Pedrini ${ }^{1}$, and Anderson Rocha ${ }^{1}$ \\ ${ }^{1}$ Institute of Computing, University of Campinas (Unicamp) \\ ${ }^{2}$ Computer Science Department, Federal University of Minas Gerais (UFMG)
}

May 19, 2015

\section{Brief Description}

Recent advances on biometrics, information forensics, and security have improved the accuracy of biometric systems, mainly those based on facial information. However, an ever-growing challenge is the vulnerability of such systems to impostor attacks, in which users without access privileges try to authenticate themselves as valid users. In our work, we present a solution to video-based face spoofing to biometric systems. Such type of attack is characterized by presenting a video of a real user to the biometric system. Our approach takes advantage of noise signatures generated by the recaptured video to distinguish between fake and valid access. To capture the noise and obtain a compact representation, we use the Fourier spectrum followed by the computation of the visual rhythm and extraction of the gray-level co-occurrence matrices, used as feature descriptors. To evaluate the effectiveness of the proposed approach, we introduce the novel Unicamp Video-Attack Database (UVAD) which comprises 17,076 videos composed of real access and spoofing attack videos. This document provides supplemental materials of the manuscript "Using Visual Rhythms for Detecting Video-based Facial Spoof Attacks" published in the IEEE Transactions on Information Forensics and Security, including instructions of how to make the download of the UVAD dataset and how to make the download of the source codes and run it in order to reproduce the results reported in the published manuscript.

\section{Download Instructions of the UVAD Dataset}

To make the download of the UVAD database, please follow the steps below:

1. Download and print the UVAD End User License Agreement, which can be found in http://figshare.com/articles/End_User_License_Agreement/1419595;

2. Sign the agreement and send it to anderson.rocha@ic.unicamp.br;

3. If your application has been approved, we will send the link to the UVAD dataset and you personal login account and password;

4. Finally, visit the UVAD website and enter your login and password information to stat the download.

\section{Download, Compile and Run Our Program from Source Code}

This program compute the noise information contained in a input video and their respective spectrum Fourier, generating a Fourier spectrum video. After, the program compute the visual rhythm from Fourier spectrum video, summarizing the most important information for face spoofing detection in a single image, named as visual rhythm.

\subsection{Requirements}

The OpenCV Package 2.4.8 is required for use this software. This software runs on Linux Operating systems, and we tested it by using Ubuntu 14.04 LTS. 


\subsection{Download the Source Code}

The program to compute the visual-rhythm maps is available on GitHub repository in the link https://github. com/allansp84/visualrhythm-antispoofing. You may to make the download of a zip file, containing the program, available on https://github.com/allansp84/visualrhythm-antispoofing/archive/master.zip or to make a clone of the repository by executing the following command:

$\$$ git clone git@github.com:allansp84/

visualrhythm-antispoofing.git.

\subsection{Compiling and Running the Program}

To compile this software the use needs set the following variables defined in the file ./Release/opencv.inc:

OPENCVFLAGS $=-\mathrm{I} / \mathrm{path} /$ to $/$ include $/$ opencv

OPENCVLIBS $=-\mathrm{L} / \mathrm{path} /$ to $/ \mathrm{lib}$

For example, suppose you have installed Opencv Package in the /home/user, then the OPENCVFLAGS and OPENCVLIBS variables should be set as:

OPENCVFLAGS = -I/home/user/include/opencv

OPENCVLIBS $=-\mathrm{L} /$ home $/$ user $/$ lib

After set the variables into ./Release/opencv.inc, the user must be run the follows commands to compile the source code:

$\$$ make -C Release clean

$\$$ make -C Release

First command line remove old binaries, and the second command builds a new binary named as ./Release/VisualRhythmAntiSpoofing.

\subsection{How to Use this Program?}

This program run only by command line interfaces (CLIs) such as the shell program (e.g., sh, bash, ksh). We provide the following parameters to the users that can be setted by the command line:

- color_space: Integer between 0 and 1 that indicates the color space used to load the video frames (default=0). Use:

○ 0: To load the frames in grayscale;

○ 1: To load the frames in the Lab color space;

- filter: Integer between 0 and 1 that indicates the type of filter used to compute the residual noise video (default $=0)$. Use:

○ 0: To use a median filter;

- 1: To use a gaussian filter.

- frame_number: Positive integer that indicates the number of consecutive frames used during computation of the visual rhythm (default $=50)$.

- input_video: Filename of the input video to be computed the visual rhythm $<$ required $>$.

- kernel_size: Positive odd integer that indicates the size of the kernel used during filtering of the input video (default $=7$ ).

- output_image: Filename of the computed visual rhythm. Visual rhythm is saved as PNG image file $<$ required $>$.

- roi_width: Positive integer that indicates the width of the region of interesting extracted of each frames (default $=30)$.

- variance: Float that indicates the variance of the Gaussian filter (default=2).

- visual_rhythm_type: Integer between 0 and 2 that indicates the type of visual rhythm to be computed from input video $<$ required $>$. Use: 
○ 0: To compute a vertical visual rhythm;

○ 1: To compute a horizontal visual rhythm;

○ 2: To compute a zig-zag visual rhythm.

Important: The parameters must be set using a hyphen (-) before the name of the parameter followed by blank space and their value (e.g., -visual_rhythm_type 0, -frame_number 50).

\subsection{Examples}

For run the program the user must be the follow parameters:

1. Compute the vertical visual rhythm of an input video (./EXAMPLE/data/testcase1.avi) using default value parameters:

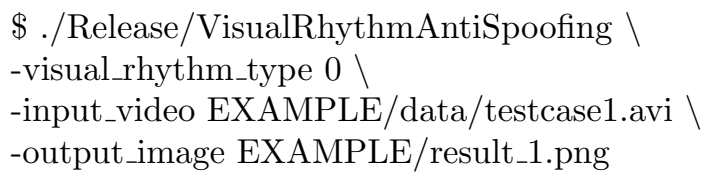

2. Compute the vertical visual rhythm from first 2 seconds of an input video (EXAMPLE/data/testcase2.avi):

\$./Release/VisualRhythmAntiSpoofing \

-visual_rhythm_type $0 \backslash$

-frame_number 50 -color_space 0 -roi_width $30 \backslash$

-filter 0 -kernel_size 7 -variance $2 \backslash$

-input_video EXAMPLE/data/testcase2.avi \

-output_image EXAMPLE/result_2.png

3. Compute the horizontal visual rhythm from first 2 seconds of an input video (EXAMPLE/data/testcase3.avi):

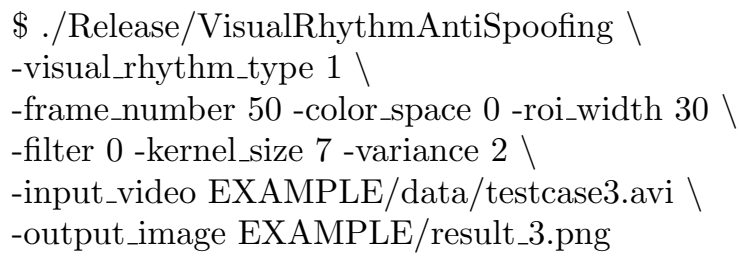

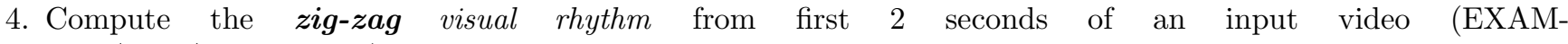
$\mathrm{PLE} /$ data/testcase1.avi):

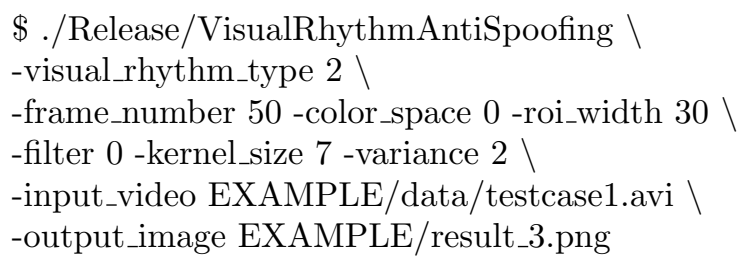

\subsection{License of this Program}

This program is available under condition of the New BSD Licence. To see the terms, please access the link https: //github.com/allansp84/visualrhythm-antispoofing/blob/master/COPYING.

\section{Reproducing the Published Results}

To reproduce the results published, please perform the following steps:

1. Download the UVAD dataset, as explained in Section 2; 
2. Download, compile and run Visual-Rhythm Antispoofing descriptor over UVAD dataset;

3. Extract texture information from visual rhythms generated by using the GLCM descritor. In the published manuscript we using implementation available in the DetectorPLS.v.0.1.1.zip, which ca be obtained in http://www.ssig.dcc.ufmg.br/detectorpls. Plase, see the DetectorPLS Manual in http://www.ssig.dcc.ufmg.br/wp-content/uploads/2014/02/manual-4.pdf to see the instruction of how to use this program. The configuration file required by the DetectorPLS program to extract the GLCM descriptor, and other descriptors evaluated in the published manuscript, can be found in:

- GLCM descritor: https://github.com/allansp84/ visualrhythm-antispoofing/blob/master/ Extra/DetectorPLS/GLCM

- LBP descriptor: https://github.com/allansp84/ visualrhythm-antispoofing/blob/master/ Extra/DetectorPLS/LBP

- HOG descriptor: https://github.com/allansp84/ visualrhythm-antispoofing/blob/master/ Extra/DetectorPLS/HOG

4. In the published manuscript, we use the PLS and SVM algorithms to classify the descriptors extracted with GLCM, LBP, and HOG methods. We use the DetectorPLS and LibSVM (version 3.18) packages to build the PLS and SVM classifiers, respectively. It is important that the evaluation criteria of the LibSVM is modified in order to use the Area Under ROC Curve (AUC) instead of accuracy (ACC), due to the unbalance of the anti-spoofing datasets. To see how to do that, please visit the link http://www.csie.ntu.edu.tw/ cjlin/libsvmtools/eval/ index.html.

\section{Please, Cite our Work!}

If you use this software, please cite our paper published in IEEE Transactions on Information Forensics and Security:

\section{Reference}

Pinto, A.; Schwartz, W.R.; Pedrini, H.; Rocha, A.d.R., "Using Visual Rhythms for Detecting Video-Based Facial Spoof Attacks," Information Forensics and Security, IEEE Transactions on , vol.10, no.5, pp.1025,1038, May 2015 doi: 10.1109/TIFS.2015.2395139

URL: http://ieeexplore. ieee.org/stamp/stamp.j.sp?tp=\&arnumber=7017526\&i snumber=7073680

\section{Bibtex}

@ARTICLE $\{7017526$,

author $=\{$ Pinto, A. and Schwartz, W.R. and Pedrini, H. and Rocha, A. $\}$,

journal $=\{$ Information Forensics and Security, IEEE Transactions on $\}$,

title $=\{$ Using Visual Rhythms for Detecting Video-Based Facial Spoof Attacks $\}$,

year $=\{2015\}$,

month $=\{$ May $\}$,

volume $=\{10\}$,

number $=\{5\}$,

pages $=\{1025-1038\}$,

keywords $=\{$ Authentication;Biometrics (access control); Databases; Face; Feature extraction; Histograms; Noise; Unicamp Video-Attack Database; Video-based Face Spoofing; Video-based face spoofing; Visual Rhythm, Videobased Attacks; impersonation detection in facial biometric systems; unicamp video-attack database; video-based attacks; visual rhythm\},

doi $=\{10.1109 /$ TIFS.2015.2395139 $\}$,

$\mathrm{ISSN}=\{1556-6013\}$,

\section{Contact}

For more information or any questions, please do not hesitate to contact us by the following email:

\section{Allan da Silva Pinto, Ph.D. Student}

e-mail: allansp84@gmail.com 